Voix et Images

voixetimages

\title{
Différents parcours de la nouvelle
}

\section{Régis Normandeau}

Volume 15, numéro 3 (45), printemps 1990

\section{Gilbert La Rocque}

URI : https://id.erudit.org/iderudit/200863ar

DOI : https://doi.org/10.7202/200863ar

Aller au sommaire du numéro

Éditeur(s)

Université du Québec à Montréal

ISSN

0318-9201 (imprimé)

1705-933X (numérique)

Découvrir la revue

Citer cet article

Normandeau, R. (1990). Différents parcours de la nouvelle. Voix et Images, 15(3), 456-458. https://doi.org/10.7202/200863ar d'utilisation que vous pouvez consulter en ligne.

https://apropos.erudit.org/fr/usagers/politique-dutilisation/ 


\section{Différents parcours de la nouvelle}

\section{par Régis Normandeau, Université du Québec à Montréal}

La nouvelle se porte plutôt bien au Québec ces dernières années: les maisons d'édition d'ici lui ont toutes fait une place dans leur catalogue; XYZ, la revue de la nouvelle se fait, quatre fois par année, depuis 1985 , le lieu de diffusion du genre et d'information sur le genre.

En constante transformation, la nouvelle québécoise ne cesse de surprendre par la diversité des thèmes et des approches auxquels elle recourt. L'année 1989 , en ce sens, n'a pas fait exception. Je vous propose ici un regard sur certaines productions qu'elle a engendrées, l'ouverture de certaines portes pour entrevoir quelques-unes des avenues de la création empruntées par les nouvelliers et nouvellières d'ici.

Le Grand Théâtre de Madeleine Ferron ${ }^{1}$, c'est celui de la vie. Les textes qui le composent mettent en scène de petites infractions au quotidien, des égratignures de l'ordre des choses. Les nouvelles se déploient avec lenteur, sans coup d'éclat; même quand il est question de pénibles face à face ("Moi, je m'appelle Hortense") ou d'échanges verbaux virulents ("La fête de famille, II"), l'écriture se déroule avec retenue, presque avec pudeur. Les finales arrivent de la même façon, presque sans qu'on s'y attende... En fait, ces nouvelles ne se terminent pas: elles restent en suspens quand l'infraction qui a donné droit d'existence à la nouvelle s'efface, laissant au lecteur l'impression qu'il n'y a pas de clôture, qu'il n'a saisi, par sa lecture, qu'une tranche d'un récit toujours en devenir. La force de ces nouvelles réside dans l'atmosphère qu'elles créent. Mais ces petites constructions cachent des réflexions qui ont une étrange résonance dans le contexte linguistique et politique actuel. Dans la nouvelle éponyme, la célèbre phrase de Claude Péloquin qui orne la murale de Jordi Bonet au Grand Théâtre de Québec inspire les mots suivants à la narratrice: Non, on n'est pas éccurés de mourir, me disais-je cyniquement, puisqu'on allonge l'agonie en pratiquant le bilinguisme plutôt que d'aller carrément à l'anglais! (p. 26) Dans "Zacharie-le-tueur", le narrateur constate: Nous pensions être des intellectuels de gauche et ne pouvions même pas discerner les vertus simples mais si nobles des gens du peuple! Il n'était pas surprenant que notre action politique soit si peu efficace! (p. 80) Ces réflexions arrivent comme des coups de hachoir dans la tranquillité de l'écriture: d'où la redoutable efficacité du message... mise en abyme dans l'efficacité de l'écriture !

France Théoret joue sur un tout autre registre dans l'Homme qui peignait Staline ${ }^{2}$. Ces récits sont des histoires de femmes. De 
tous âges, de toutes conditions. Mais ici, le quotidien est très différent de celui décrit par Madeleine Ferron. France Théoret se livre à une véritable introspection du vécu de ses personnages, de leurs questionnements. Chaque attitude, chaque sentiment est disséqué au scalpel des attentes déçues. Le rythme est lent, sans cesse sollicité par la négociation que ces femmes mènent entre leurs espoirs et la réalité. Les textes sont denses, porteurs de réflexions sur les relations qu'entretiennent les femmes avec autrui. Dans la nouvelle éponyme, Louise Aubert construit une relation amoureuse tout en assumant son héritage social et familial. D'autres nouvelles nous dépeignent une femme qui doit conjuguer une relation de couple et une volonté d'apprivoisement des mots ("Sans visage»), une autre qui assume pleinement sa décision de rompre une relation vieille de douze ans. Mais le texte le plus dense est, à mon avis, "Le tweed anglais" (Voix \& images en a publié un extrait dans le $n^{\circ} 40$ ) qui met en scène une mère de famille nombreuse - rôle traditionnel par excellence - qui tient tête à un mari joueur. Elle doit réprimer ses rêves de jeunesse ( $[. .$. ) elle ne sera pas la femme que son désir n'est jamais parvenu à préciser), sollicitée qu'elle est par son rôle de mère, [...] désorientée par sa propre fécondité [...]. Elle met tout son espoir dans le symbolisme représenté par le tweed avec lequel elle veut fabriquer des vêtements pour ses filles, symbole de classe. Espoir qui sera aussi déçu. Tout cela sous le regard de la fille aînée qui commence à comprendre ce qui se passe, mais n'ose demarider des précisions. Cette nouvelle condense à peu près tout ce qui essaime dans les autres textes du recueil: présence obsédante du passé, quête d'un avenir meilleur $([\ldots .$.$] des malheurs plus grands peuvent$ survenir), relation avec un homme, prise en charge de son destin. D'où sa puissance d'évocation.

Aérographies ${ }^{3}$ va dans une tout autre direction: l'interrelation entre deux médiums artistiques. Cinq auteurs ont été conviés à écrire une nouvelle à partir d'une ouvre picturale de Jean-Pierre Neveu exécutée selon la technique de l'aérographe. Donc ici, ce 'n'est pas l'écrit qui engendre le pictural, mais l'inverse: l'image se laisse dire, suscite le travail de la parole. Les rapports qui se tissent alors entre l'image et les mots sont très variés d'un auteur à l'autre, différentes caractéristiques de l'image les sollicitant. André Berthiaume ("Conte bleu ") se laisse séduire par la forme - plutôt l'informe - même et la couleur bleue qui caractérisent le tableau (l'Interprète du silence) pour en tirer une forme de vie primitive vue au microscope. DianeMonique Daviáu («Les inséparables", tiré des Inséparables) s'attache à la précision des formes et à leur couleur pour tisser un texte angoissant où vivent des saccageurs de sommeil (p. 32). Daniel Sernine ("Sur la Jetée») et Marie José Thériault ("Reviendrons-nous enfin à Saint-Gilles à la nuit tombante?"), quant à eux, tissent des relations plutôt abstraites entre l'image et l'écrit: le premier tire 
d'une représentation du big bang (Naissance du temps, reproduite en page couverture) l'hallucinante histoire d'un séjour dans une étrange pièce, alors que la deuxième tire de Chez nous partout le récit de la consolidation d'un amour à travers un voyage. André Vanasse, enfin, fait une "lecture au pied de la lettre (de limage?)" du tableau la Poursuite et concocte l'histoire d'un oto-rhino-laryngologiste dont la découverte d'une gorge très particulière bouleverse la vie. À cette relation nouée entre les cinq auteurs et les tableaux, s'ajoutent les titres que Jean-Pierre Neveu a donnés à ses œuvres: ils constituent une première lecture, un pré-texte traité différemment par les auteurs: complètement assumé par Diane-Monique Daviau, complètement occulté par André Vanasse, intégré à divers degrés par les trois autres auteurs. Donc, ces cinq représentations cosmiques (p. 7) inspirent cinq textes très différents. Il faut aussi souligner ce très joli nom que porte la collection, "Pictographe", et qui rend très bien compte à la fois de l'intention et de la facture de l'ouvrage.

Ces trois recueils sont exemplaires de la production nouvellière récente: diversité des thèmes, du ton, de la facture. Ils ne constituent qu'un très mince échantillon d'un genre de plus en plus prisé par les auteurs québécois.

1 Madeleine Ferron, le Grand Théâtre, Montréal, Boréal, 1989, 152 p.

2 France Théret, l'Homme qui peignait Staline, Montréal, les Herbes rouges, $1989,174 \mathrm{p}$.

3 André Vanasse (directeur), Aérographies, Montréal, XYZ éditeur, 1989, 78 p. (Pictographe). 